\title{
A Fourier iterative method for solving axi-symmetric integral equations
}

\author{
Allen Q. Howard, Jr., Universidade Estadual \\ do Norte Fluminense Darcy Ribeiro
}

Copyright 2016, SBGf - Sociedade Brasileira de Geofísica.

Este texto foi preparado para a apresentação no VII Simpósio Brasileiro de Geofísica, Ouro Preto, 25 a 27 de outubro de 2016. Seu conteúdo foi revisado pelo Comitê Técnico do VII SimBGf, mas não necessariamente representa a opinião da SBGf ou de seus associados. É proibida a reprodução total ou parcial deste material para propósitos comerciais sem prévia autorização da SBGf.

\section{Abstract}

This paper presents a new iterative method for solving axi-symmetric integral equations. The theory is developed for a two-dimensional axisymmetric inhomogeneous medium. Here to test the accuracy and efficiency of the method, a simple onedimensional layered medium geometry is assumed. In this case the problem reduces to sequences of one-dimensional convolutions using fast Fourier transforms along the axial direction $z$. The equation is renormalized to help insure that the Neumann series iterations converge, even in the limit of high contrasts. The transforms are designed to mimic their continuous counterparts on semi-infinite intervals. Validation includes comparisons with wellknown analytical results for layered earth models.

\section{Introduction}

Integral equation solutions using finite-element or moment methods result in full matrices usually limiting such methods to cases where relative anomaly volumes are small. For linearized solutions, the convolutional form of the integral equation is often exploited by Fourier transforming the equation into the spatial frequency domain to perform the convolution between the Green's function and the volume current using FFT's (fast Fourier transforms). The integral equation naturally results in order 1 Fourier-Bessel transforms. These transforms however are not directly amenable to Cartesian coordinate FFT computation.

A form of the integral equation this method is designed to solve is given by(Howard, 2014). This type of equation is used to model induction logging tool response in borehole geophysical applications(Chew,1991). In order to realize the time savings fast Fourier transform operation count proportional to $N \log _{2} N$, it is necessary to keep the computation in memory. For three-dimensional problems, work stations can provide this type of CPU memory resource (perhaps 128 to $256 \mathrm{~GB}$ ). In the case of axial-symmetry, the integral equation for the electric field component $\mathrm{e}_{\phi}(\mathbf{x})$ is pseudo-scalar and is shown to take the form

$$
\mathrm{e}_{\phi}(\mathbf{x})=\mathrm{e}_{\phi, 0}(\mathbf{x})+\frac{1}{2 \pi} \int g_{1}\left(\mathbf{x}, \mathbf{x}^{\prime}\right) j\left(\mathbf{x}^{\prime}\right) \mathrm{d}^{3} x^{\prime}
$$

where the volume current $j(\mathbf{x})$ depends on the unknown electric field $\mathrm{e}_{\phi}(\mathbf{x})$ and is defined in terms of the profile function $p(\mathrm{x})$, i.e.,

$$
\begin{aligned}
& j(\mathbf{x})=p(\mathbf{x}) \mathrm{e}_{\phi}(\mathbf{x}), \\
& p(\mathbf{x})=k^{2}(\mathbf{x})-k_{b}^{2},
\end{aligned}
$$

and the integration is over all space. The azimuthally symmetric Green's function in equation (2) for a loop source transmitter is given by

$$
g_{1}\left(\mathbf{x}, \mathbf{x}^{\prime}\right)=\int_{0}^{2 \pi} \cos \left(\phi-\phi^{\prime}\right) \frac{e^{i k_{b} R}}{4 \pi R} \mathrm{~d} \phi^{\prime}
$$

where $R=\left|\mathbf{x}-\mathbf{x}^{\prime}\right|$ and $k_{b}$ is the intrinsic quasi-static electromagnetic background wavenumber in units of $\left[\mathrm{m}^{-1}\right]$. The known background electric field $\mathrm{e}_{\phi, 0}(\mathbf{x})$ is the solution to the homogeneous space problem with wavenumber $k_{b}=$ $\left(i \omega \mu_{0} \sigma_{b}\right)^{1 / 2}$ with $\operatorname{Im}\left(\mathrm{k}_{\mathrm{b}}\right) \geq 0$ for a time factor of $e^{-i \omega t}$. Here $\sigma_{b}[\mathrm{~S} / \mathrm{m}]$ is the earth model background conductivity, $\mu_{0}=$ $4 \pi \times 10^{7}[\mathrm{H} / \mathrm{m}]$ is the magnetic permeability of free space, $\omega=2 \pi f$, and $f$ is the transmitter frequency in $\mathrm{Hz}$. For this analysis we use the Green's function $g_{1}\left(\mathbf{x}, \mathbf{x}^{\prime}\right)$ in the form (howard,2014), p. 11

$$
g_{1}\left(\mathbf{x}, \mathbf{x}^{\prime}\right)=\int_{-\infty}^{\infty} \mathrm{d} K_{z} e^{i K_{z}\left(z-z^{\prime}\right)} \int_{0}^{\infty} \mathrm{d} K_{\rho} K_{\rho} \frac{J_{1}\left(K_{\rho} \rho\right) J_{1}\left(K_{\rho} \rho^{\prime}\right)}{K^{2}-k_{b}^{2}}
$$

Note that the integral equation defined by equation (1) is a convolution of the Green's function and the volume current $j\left(\mathbf{x}^{\prime}\right)$. Substitution of representation (4) into integral equation (1) thus gives the transformed integral equation for an axi-symmetric 2D formation

$$
\mathrm{E}\left(K_{\rho}, K_{z}\right)=\mathrm{E}_{0}\left(K_{\rho}, K_{z}\right)+\mathrm{G}(\mathrm{K}) \mathrm{J}\left(K_{\rho}, K_{z}\right),
$$

where

$$
\mathrm{G}(\mathrm{K})=1 /\left(K^{2}-k_{b}^{2}\right)
$$

and $K^{2}=K_{\rho}^{2}+K_{z}^{2}$ and where

$$
\mathrm{E}^{(n)}\left(K_{\rho}, K_{z}\right)=\int_{-\infty}^{\infty} e^{-i K_{z} z} \mathrm{~d} z \int_{0}^{\infty} \rho \mathrm{J}_{1}\left(K_{\rho} \rho\right) e_{\phi}^{(n)}(\rho, z) \mathrm{d} \rho, \quad n=0,1, \cdots
$$

The function $\mathrm{J}\left(K_{\rho}, K_{z}\right)$ is the transform of the volume current defined as 


$$
\mathrm{J}\left(K_{\rho}, K_{z}\right)=\int_{-\infty}^{\infty} e^{-i K_{z} z^{\prime}} \mathrm{d} z^{\prime} \int_{0}^{\infty} \rho^{\prime} \mathrm{J}_{1}\left(K_{\rho} \rho^{\prime}\right) j\left(\rho^{\prime}, z^{\prime}\right) \mathrm{d} \rho^{\prime},
$$

with analogous transform definitions for the background and total electric fields. It is interesting and gratifying to note that the form of integral equation given by (5) or (12) is the same as that for the scalar field $2 \mathrm{D}$ equation given by equations (7.11), (7.12) and (7.13) in (howard, 2014).

The more simple one-dimensional layered medium case where the profile varies only in the $z$ direction $(p(\mathbf{x})=p(z))$ results in the simplification

$$
\mathrm{J}\left(K_{\rho}, K_{z}\right)=P\left(K_{z}\right) * E\left(K_{\rho}, K_{z}\right) .
$$

In this case, a more explicit form of the integral equation (5) in $\left(K_{\rho}, z\right)$ space is

$$
\mathrm{E}\left(K_{\rho}, z\right)=\mathrm{E}_{0}\left(K_{\rho}, z\right)+\int_{-\infty}^{\infty} \mathrm{d} z^{\prime} \frac{e^{-\gamma\left|z-z^{\prime}\right|}}{2 \gamma} \mathrm{E}\left(K_{\rho}, z^{\prime}\right) p\left(z^{\prime}\right) .
$$

where

$$
\gamma=\left(K_{\rho}^{2}-k_{b}^{2}\right)^{1 / 2}, \quad \operatorname{Rl}(\gamma) \geq 0 .
$$

In the case of a two coil induction sonde with transmitter and receiver coils separated by the axial distance $L$, i.e. $Z_{T}=Z_{R}+L$, the induction log reponse over the entire formation defined by the profile $p(z)$ can be computed by convolution. In fact, an iterative solution to the $1 \mathrm{D}$ integral equation (10) is simply, at each iteration, the convolution

$$
\mathrm{E}^{(n)}\left(K_{\rho}, z\right)=\mathrm{E}_{0}\left(K_{\rho}, z\right)+\mathrm{G}\left(\mathrm{K}_{\rho}, z\right) *\left(\mathrm{E}^{(n-1)}\left(\mathrm{K}_{\rho}, z\right) p(z)\right),
$$

for $n=1,2, \cdots$, beginning with initial estimate $E^{(0)}\left(K_{\rho}, K_{z}\right)=$ $E_{0}\left(K_{\rho}, K_{z}\right)$.

To enhance the iterative convergence of the Neumann series solution, equation (12) is rewritten in the renormalized form

$$
\begin{aligned}
\mathrm{E}^{(n)}\left(K_{\rho}, z\right)= & \mathrm{N}\left(K_{\rho}, z\right) \mathrm{E}_{0}\left(K_{\rho}, z\right)+\mathrm{N}\left(K_{\rho}, z\right)\left[\mathrm{G}\left(\mathrm{K}_{\rho}, z\right) *\right. \\
& \left.\left(\mathrm{E}^{(n-1)}\left(\mathrm{K}_{\rho}, z\right) p(z)\right)-\mathrm{M}\left(K_{\rho}\right) \mathrm{E}^{(n-1)}\left(K_{\rho}, z\right)\right], \\
& n=1,2,3 \cdots .
\end{aligned}
$$

where

$$
\begin{aligned}
& \mathrm{N}\left(K_{\rho}, z\right)=1 /\left(1-\mathrm{M}\left(K_{\rho}, z\right)\right), \\
& \mathrm{M}\left(K_{\rho}, z\right)=\mathrm{G}\left(K_{\rho}, z\right) * p(z) .
\end{aligned}
$$

Beginning with the known starting point $E^{(0)}\left(K_{\rho}, z\right)=$ $E_{0}\left(K_{\rho}, z\right)$, the idea is to iteratively solve the onedimensional integral equation (13) for $E\left(K_{\ell}, z\right) N_{r}$ times, one for each quadrature abscissa $K_{\ell}, \ell=1,2, \cdots, N_{r}$ and then, after the iterative procedure, compute the solution to the integral equation by numerical quadrature of the Fourier Bessel- transform, i.e.

$$
\mathrm{e}_{\phi}(\rho, z)=\sum_{\ell=1}^{N_{r}} J_{1}\left(K_{\ell} \rho\right) K_{\ell} E\left(K_{\ell}, z\right) w_{\ell} .
$$

Quite generally, to consider the numerical convergence of the iterative solution to integral equation (1), rewrite it in terms of the linear integral operator L, i.e.,

$$
\mathrm{e}_{\phi}(\mathbf{x})=\mathrm{e}_{\phi, 0}(\mathbf{x})+\mathrm{Le}_{\phi}(\mathbf{x})
$$

Then formally, the iterative solution, if it exists, takes the form

$$
\begin{aligned}
\mathrm{e}_{\phi}(\mathbf{x}) & =\mathrm{e}_{\phi, 0}(\mathbf{x})+\mathrm{Le}_{\phi, 0}(\mathbf{x})+\mathrm{L}^{2} \mathrm{e}_{\phi, 0}(\mathbf{x})+\cdots, \\
& =[\mathrm{I}-\mathrm{L}]^{-1} \mathrm{e}_{\phi, 0}(\mathbf{x})
\end{aligned}
$$

A necessary condition for the convergence of the Neumann series solution given by equation (17) is that the norm of the operator be less than one, i.e.,

$$
|\mathrm{L}|<1 .
$$

The convergence depends on the profile function $p(\mathbf{x})$ and the singularity of the Green's function $g_{1}\left(\mathbf{x}, \mathbf{x}^{\prime}\right)$. Habashy (Habashy,1993), by modifying the equation, softens the singularity in the Green's function, resulting in a simple method to enhance convergence. His idea is to modify the defining equation (1) by adding and subtracting the term $\frac{1}{2 \pi} \mathrm{e}_{\phi}(\mathbf{x}) \int g_{1}\left(\mathbf{x}, \mathbf{x}^{\prime}\right) p\left(\mathbf{x}^{\prime}\right) \mathrm{d}^{3} x^{\prime}$ and thus obtain a form of the integral equation more amenable to Neumann iteration, e.g.,

$$
\begin{aligned}
\mathrm{e}_{\phi}(\mathbf{x})= & n(\mathbf{x}) \mathrm{e}_{\phi, 0}(\mathbf{x})+n(\mathbf{x}) \frac{1}{2 \pi} \int g_{1}\left(\mathbf{x}, \mathbf{x}^{\prime}\right) p\left(\mathbf{x}^{\prime}\right) \\
& \left(\mathrm{e}_{\phi}(\mathbf{x})-\mathrm{e}_{\phi}\left(\mathbf{x}^{\prime}\right)\right) \mathrm{d}^{3} x^{\prime},
\end{aligned}
$$

where the re-normalization function is defined as

$$
n(\mathbf{x})=1 /(1-m(\mathbf{x})),
$$

and where the associated normalization function is

$$
m(\mathbf{x})=\frac{1}{2 \pi} \int g_{1}\left(\mathbf{x}, \mathbf{x}^{\prime}\right) p(\mathbf{x}) \mathrm{d}^{3} x^{\prime} .
$$

A stair-case one-dimensional layered medium profile function $p(z)=k^{2}(z)-k_{b}^{2}$ for $M$ regions with ordered bedboundaries $z_{m}$, such that $z_{m}>z_{n}$ when $m>n$ and for $M>2$ can be written in the form

$$
p(z)=k_{1}^{2} u\left(z_{1}-z\right)+\sum_{m=2}^{M-1} \operatorname{rect}\left(\frac{z-d_{m}^{(+)}}{2 d_{m}^{(-)}}\right) k_{m}^{2}+u\left(z-z_{M}\right) k_{M}^{2}-k_{b}^{2},
$$




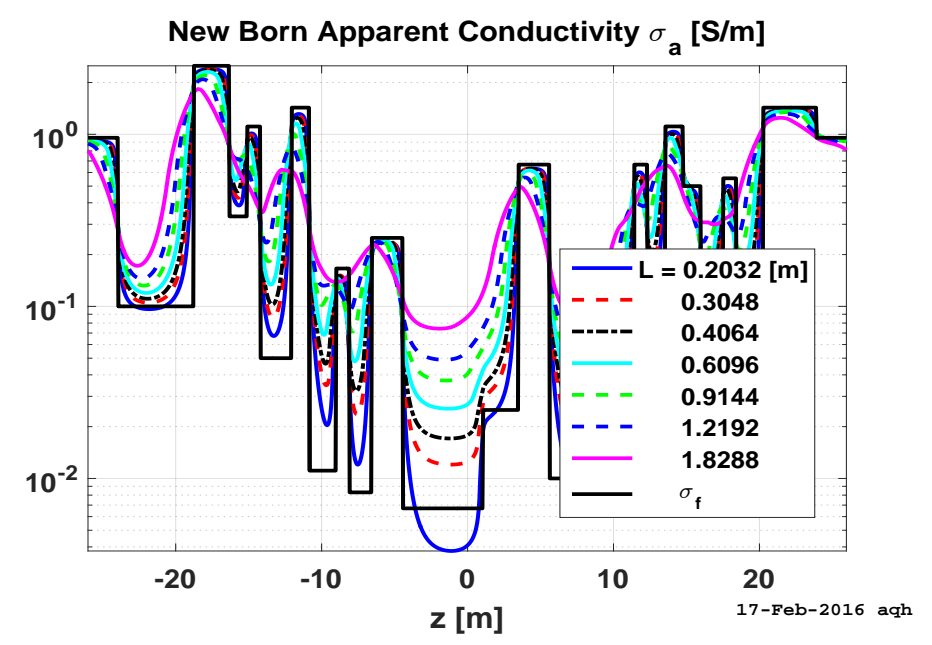

Figure 1: Two-coil Apparent Conductivity $\sigma_{a}$ for the array defined in the legend as a function of $z$.

where the step function $u(z)$ is defined as

$$
u(z)= \begin{cases}1, & z \geq 0 \\ 0, & \text { otherwise }\end{cases}
$$

The iterative model defined by equation (12) is a generalization of the linearized mode developed I in (Howard,1986) and (Howard,1987). Beginning with the known starting point $E^{(0)}\left(K_{\rho}, z\right)=E_{0}\left(K_{\rho}, z\right)$, as given by equation (14), the idea is to iteratively solve the onedimensional integral equation (12) for $E\left(K_{\ell}, z\right) N_{r}$ times, one for each quadrature abscissa $K_{\ell}, \ell=1,2, \cdots, N_{r}$ and then compute the solution to the integral equation by numerical quadrature of the Fourier Bessel- transform, i.e.

$$
\mathrm{e}_{\phi}(\rho, z)=\sum_{\ell=1}^{N_{r}} J_{1}\left(K_{\ell} \rho\right) K_{\ell} E\left(K_{\ell}, z\right) w_{\ell}
$$

where $w_{\ell}$ is the quadrature weight coefficient associated with abscissa $K_{\ell}$.

\section{Preliminary check of apparent conductivity}

A relatively simple preliminary check on the formulation is to compute the first iteration of equation (12). This results in a form of the Born approximation for the total electric field. For numerical comparisons we define a two-coil induction array with coil spacings of $L=$ $0.2032,0.3048,0.4064,0.6096,0.9144$, and 1.2192, 1.8288 [m]. For this computation, the total electric field is scaled in traditional apparent conductivity $\left(\sigma_{a}(z)\right)$ units.

The family of apparent conductivities shown in Fig. 1 are as expected with the higher vertical resolution shorter arrays tracking more closely the actual input formation conductivity $\sigma_{f}$ near the bed-boundary transitions.

Figure ?? is the large dynamic range one-dimensional 28 layer Oklahoma input formation conductivity profile $\sigma(z)$ $[\mathrm{S} / \mathrm{m}]$ used in the simulations to follow. The background conductivity is $\sigma_{b}=0.9545[\mathrm{~S} / \mathrm{m}]$. Table ?? defines other input parameters for the simulation. Figure ?? compares the computation of equation (??) as compute by 256 point Gauss-Legendre quadrature with an FFT numerical convolution with respect to $z$.

\section{Conclusions}

The application of the results of this as well as the companion paper is to develop an efficient numerical method to compute three-dimensional convolutions. For axi-symmetric geometries, this is an important component for efficient iterative solutions to integral equations. In this paper Fourier-Bessel transforms of order one, that use the discrete sine and cosine transforms developed in the companion paper, are validated by comparing the computations with analytic examples of Green's functions and an associated normalization function. Optimum computation speed of the resulting three-dimensional solver depends upon keeping all arrays in memory.

\section{Acknowledgements}

I thank my host, Professor A. Abel G. Carrasquilla at UENF, Macaé, for providing the opportunity and encouragement to participate again in applied geophysics in Brazil.

\section{References}

Howard, A.Q., Jr., Fourier iterative method for induction logging applications, VI Simpósio Brasileiro de Geofísica, October 14-16, (4583), 2014.

Chew, W. C., S. Barone, B. Anderson, and C. Hennesy, Diffraction of axi symmetric waves in a borehole by bed boundary discontinuities, Geophysics, vol 49, no. 10, 1984.

Chew, W. C., Z. Nie, Q-H. Liu and B. Anderson, An efficient solution for the response of electrical well logging tools in a complex environment, IEEE Trans. on Geoscience and Remote Sensing, vol 29, no. 2, 1991.

Gao, G. and C. Torres-Verdín, Efficient Numerical Simulation of Axisymmetric Electromagnetic Induction Measurements Using a High-Order Generalized Extended Born Approximation, IEEE Transactions on Geoscience and Remote Sensing, vol 44, no 9, 2006.

Habashy, T. M., R. A. Groom and B. R. Spies, Beyond the Born and Rytov Approximations: A Nonlinear Approach to Electromagnetic Scattering, vol 98, no 82, 1993.

J. A. Ferrari, Fast Hankel transform of order zero, JOSA Communications, vol. 12, no. 8, 1995.

D. R. Mook, An algorithm for the numerical evaluation of the Hankel and Abel Transforms, IEEE Transactions on acoustics, speech, and signal processing, vol 31, no. 4, 1983. 